\title{
LOS VALORES EN EDUCACIÓN PARA EL DESARROLLO ${ }^{1}$
}

VALUES IN EDUCATION FOR DEVELOPMENT

\author{
Juan Manuel Zamora Mendoza ${ }^{2}$ \\ Miguel Ángel Medina Romero ${ }^{3}$ \\ Jean Cadet Odimba OnÉtambalako Wetshokonda ${ }^{4}$
}

Para citar este artículo: Zamora, J., Medina, M. y OnEtambalako, J. (2014). "Los valores en educación para el desarrollo”. Inquietud Empresarial. Vol. XIV (2), p.p 155-170

Fecha de Recepción: 13 de agosto de 2014

Fecha de Aceptación: 26 de noviembre de 2014

\begin{abstract}
1 Artículo de Reflexión como resultado del proyecto de investigación titulado "Agenda digital para el Desarrollo del Estado de Michoacán: Planteamiento y Construcción de las Bases Institucionales y Jurídicas de una Sociedad de la Información del siglo XXI en la coordinación de Investigación Científica de la UMSNH".

2 Doctor en Derecho por la Universidad de Guadalajara; Maestro en Derecho y Licenciado en Derecho por la Universidad Michoacana de San Nicolás de Hidalgo; profesor e investigador Titular "B", tiempo completo, perfil PROMEP-SEP; Consejero Universitario, Consejero Técnico, Cuerpo Académico "Derecho y Ciencias Sociales" UMSNH-CA-205 Consolidado, miembro del Centro de Investigaciones Jurídicas y Sociales, miembro de la Red Internacional de Investigadores en Competitividad; profesor e investigador en licenciatura y Posgrado Facultad de Derecho y Ciencias Sociales de la Universidad Michoacana de San Nicolás de Hidalgo. manueljz49@gmail.com

3 Doctor en Derecho, Sistema Nacional de Investigadores (SIN), perfil PROMEP-SEP, Cuerpo Académico "Derecho y Ciencias sociales" UMSNH-CA-205 Consolidado, Miembro del Centro de investigaciones Jurídicas y Sociales, miembro de la Red Internacional de Investigadores en Competitividad, actualmente, profesor e investigador en licenciatura y posgrado Facultad de Derecho y Ciencias Sociales Universidad Michoacana de San Nicolás de Hidalgo.miguel_medina_romero@hotmail.com 4 Doctor en Derecho, Sistema Nacional de Investigadores (SIN), perfil PROMEP-SEP, Cuerpo Académico "Derecho y Ciencias sociales" UMSNH-CA-205 Consolidado, Miembro del Centro de investigaciones Jurídicas y Sociales, actualmente, profesor e investigador en licenciatura y posgrado Facultad de Derecho y Ciencias Sociales Universidad Michoacana de San Nicolás de Hidalgo. jcodimba@ hotmail.com
\end{abstract}




\title{
RESUMEN
}

Referenciar a la dimensión axiológica de la educación como estrategia de desarrollo significa explicitar qué sistema valorativo se asume. Es importante poner el centro en la dimensión axiológica por varias razones: primero, porque toda persona sea buena o mala y antes de serlo es forzosamente moral, por ser inteligente y libre. Moral, como estructura porque tiene que justificar su vida, en su totalidad y, acto por acto, en cada una de sus partes; como contenido, porque el ser humano es libre y tiene que elegir cuál va a ser su bien, de realizarlo en el mundo y de apropiárselo personalmente. No existe el hombre biológico, desnudo de cultura, de valores, estamos llamados a recorrer ese camino, a cuestionarnos lo que nos rodea, a valorar y actuar en consecuencia. Segundo, porque tanto en el ámbito de la educación como en el del desarrollo, se refleja el carácter moral del ser humano. En educación, la condición moral se manifiesta en la actuación profesional, en los contenidos educativos; en cualquier actuación profesoral estamos filtrando y proyectando una determinada concepción de la persona, promoviendo unos determinados valores, por lo mismo, no podemos renunciar a nuestra condición de humanos que viven y actúan desde unos valores.

\section{PALABRAS CLAVE}

Valores, educación, persona, estrategia, desarrollo.

\begin{abstract}
Referencing the axiological dimension of education as a development strategy means explain what value system we take. It is important to put the center in the axiological dimension for several reasons: first, because every person is good or bad and before is necessarily be moral, to be intelligent and free. Moral like structure that has to justify his life in its entirety and, act by act, in each of its parts; as content, because human beings are free and have to choose what will be your good, do it in the world and appropriate it personally. There is no biological man, naked from the culture, values, are called to follow that path, to question what is around us, to value and act accordingly. Second, both in education and in the development of the moral nature of man is reflected. In education, the moral condition is manifested in the performance, in school curricula; at any professorial performance we are filtering and projecting a certain conception of the person promoting certain values, therefore, we cannot give up our humanity to live and act from values.
\end{abstract}

\section{KEYWORDS}

Values, education, person, strategy, development. 


\section{INTRODUCCION}

Los valores morales en la educación. No se puede olvidar el componente moral de la educación. Si la condición de ser humano como tal le obliga a ejercitar continuamente la capacidad de discernir entre lo bueno o lo malo, lo mejor y lo peor, y si la educación ha de preparar para la vida, no se puede, por tanto, dejar a un lado lo que constituye la esencia misma de la persona (Payá, 1997).

El que se propone en esta reflexión no es un modelo moral absoluto, pero sí que incorpora los valores morales contenidos en la Declaración Universal de los Derechos Humanos como orientación para la resolución de conflictos morales individuales y colectivos. En palabras de Bobbio (1991), la declaración Universal de los Derechos Humanos puede ser aceptada como la mayor prueba histórica que nunca han existido del consensum ómnium gentum de un sistema de valores. En esta misma línea, el jurista An-Na'lm (1992; 1999), afirma que se ha conseguido un grado de consenso universal acerca de los derechos humanos, que incluye los derechos de las tres generaciones. Una de las críticas más poderosas a la pretendida universalidad de los derechos humanos es la que proviene del relativismo cultural. Los defensores de esta línea argumentan que los valores y las prácticas culturales son características de cada cultura, y que cada una de ellas tiene el derecho de ser diferente, distinta y única (Howard, 1993). Para profundizar en los aspectos de esta polémica y en las vías de consenso entre ambas posturas, puede consultarse Monzón (1992) y De Lucas (1994).

Pero ¿Qué son los valores morales? ¿Qué los diferencia de otro tipo de valores, como los estéticos, los religiosos, los económicos? Según la propuesta de Siurana (1998), los valores morales tienen tres características fundamentales: 1) sólo se aplican a personas, 2) el asumirlos como propios depende de la libertad, y 3) se consideran válidos para todas las personas. Asimismo, afirma que los valores morales son prescriptivos, perdurables y dan coherencia a nuestras vidas.

Según Pérez (1996), los valores morales señalan un deber-ser que el hombre se establece para sí mismo. Ese deber-ser se traduce a veces en normas morales con carácter obligatorio, cuando se refiere a las cuestiones de justicia, y otras veces se perfila sólo a través de criterios de valoración que permiten enjuiciar la calidad humana de nuestras realizaciones. Este es el campo de las cuestiones relativas a la autorrealización o felicidad de las personas.

Siguiendo a Camps (1994), los valores morales que emanan de la declaración Universal de los Derechos Humanos y que pueden ser tomados como valores guía para una propuesta de educación en valores son los siguientes: dignidad, libertad, igualdad, responsabilidad, diálogo, respeto activo-tolerancia, solidaridad, justicia y paz. Esta afirmación sería muy discutida por los defensores de un enfoque sociologista reduccionista, que entienden que todos los fenómenos morales están generados por condiciones sociales y son consecuencia de sanciones sociales sobre la conducta. Para profundizar en la relación entre sociología y valores puede consultarse el trabajo de Giner (1989).

\section{La dignidad}

El artículo tercero de la Declaración Universal de Derechos Humanos afirma que todo individuo tiene derecho a la vida, a la libertad y a la seguridad de su persona. El derecho a la vida significa el derecho a no verse privado de la vida por la voluntad arbitraria de otros o de los poderes establecidos. Tras el derecho a la vida está el derecho a la libertad, ya que no es comprensible una vida auténticamente humana 
privada de libertad. La libertad es la que dota a la vida humana de una dignidad especial. Por otro lado, el derecho a la seguridad implica el poder moverse y actuar con libertad sin que su vida peligre por ello (Camps, 1994).

Por otro lado, en el segundo imperativo categórico kantiano está contenida la esencia de la dignidad humana: "Obra de tal modo que uses la humanidad, tanto en tu persona como en la persona de cualquier otro, siempre como un fin al mismo tiempo y nunca solamente como un medio" (Kant, 1992) o en palabras de Camps (1994), "piensa que el otro es tan persona como tú y trátalo como tal persona y no como una cosa susceptible de estar sólo a tu servicio".

\section{La libertad}

Constant (1989) dice, el valor de la libertad tiene diferentes acepciones; podemos de la libertad como autonomía o libertad positiva, de libertad negativa o libertad de los modernos y de libertad como participación o libertad de los antiguos.

La libertad como autonomía es un valor que nace en la ilustración. Kant (1992) habla de un ser humano autónomo que es capaz de darse a sí mismo las leyes que van a regular su vida. Es un valor estrechamente ligado a la formación de la conciencia moral. La libertad como autonomía puede ser asimilada a lo que Nussbaum (1999) define como razón práctica: "todos los seres humanos participan -o intentan- en planificar y dirigir sus propias vidas, haciendo y respondiendo preguntas acerca de lo que es bueno y cómo debería uno vivir. Además, desean realizar sus ideas en sus vidas, ser capaces de elegir y evaluar y funcionar de acuerdo con ellas" (1998).

Por su parte, Berlín (1988) asimila el concepto de libertad como autonomía a la libertad positiva, entendida como el deseo por parte del individuo de ser su propio dueño. La idea ha sido una idea clave para articular morales cosmopolitas y universalistas, ya que, como sugiere Thiebaut (1999), la lógica de la autonomía nos supone a todos y a cada uno fuente válida de demandas normativas y tribunal último de nuestra racionalidad, a pesar de otras consideraciones puedan sospechar que no somos libres, que estamos engañados o que perseguimos ocultos intereses.

La idea de libertad como autonomía o libertad positiva están muy ligadas a las teorías sobre el desarrollo del juicio moral. Al analizar las teorías de Kohlberg (1992), tener conciencia moral significa ir ganando una mayor autonomía, hacer lo que hay que hacer por convicción, de manera libre, y no por imposición.

Una segunda acepción del término libertad es la libertad negativa, según la definición de Berlín (1988), o libertad de los modernos según Constant (1989). Este concepto surge en los siglos XVI y XVII y se asimila a la libertad como independencia. En su definición original, consistía en garantizar un ámbito en el que nadie podía interferir, en el goce apacible de la independencia privada y en la renuncia a la libertad de los antiguos, a la participación activa en el poder colectivo, pues eso puede conducir a someter el individuo a la comunidad. La libertad negativa coincide con las libertades civiles y políticas, libertad para decidir lo que uno piensa, para asociarse con quien uno quiera, para votar o dejar de hacerlo.

El tercer significado del valor libertad, es libertad como participación o libertad política. Es lo que Constant (1989) denominó libertad de los antiguos. Según Peces (1999), la libertad como participación consiste en la libertad para intervenir en los criterios de decisión política; está en la raíz de los derechos fundamentales que permiten contribuir al proceso de producción normativa, a las manifestaciones 
de la voluntad del Estado, y al establecimiento de fines, objetivos y valores de su actividad. Pero, a nuestro juicio, la libertad como participación no tiene que limitarse únicamente a la vida política, sino que ha de extenderse a otros ámbitos de la vida social en los que también se toman decisiones que nos afectan (Martínez, 2000). Por ello, deben abrirse causes reales y variados de posible participación para que todos puedan intervenir en la deliberación y toma de decisiones que les afectan.

\section{La igualdad}

La Declaración Universal de los Derechos Humanos afirma que los seres humanos son iguales en dignidad y derechos, de tal manera que, por un lado, se afirma el principio de la ley natural de que todos los hombres nacen iguales y libres y, por otro, se sugiere que los derechos humanos enunciados en la Declaración deben constituir un mínimo común denominador de las legislaciones de todos los países.

Bobbio ( 1991) sugiere un interesante paralelismo entre la evolución del principio de igualdad y el de libertad. Así, a la idea de libertad negativa corresponde el momento de la igualdad jurídica o igualdad de todos los ciudadanos ante la ley. A todos los ciudadanos se les reconoce capacidad jurídica, lo que implica una abstracta capacidad de querer y actuar, en los límites de las leyes, en el propio interés. Por otro lado, a la libertad política o libertad de participación se corresponde con la igualdad política, es decir, la igualdad de todos los ciudadanos para participar en las decisiones políticas. Por último, la libertad positiva o libertad como autonomía se relaciona con la igualdad social, también denominada igualdad material o igualdad de oportunidades. Es éste el valor que fundamenta los derechos económicos y sociales (los llamados derechos de segunda generación) y que presupone que la garantía de estos derechos es condición indispensable para poder ejercer la libertad. El modelo del Estado de bienestar es la aportación política más definitiva a favor de la igualdad. Como sostiene Petrella, "con la creación del Estado Welfare, la redistribución de la riqueza es, por primera vez, decididas, establecida y acordada por el conjunto de la población de un país (...) a favor de los ciudadanos (1997). Este autor sostiene que el Estado del Bienestar ha ido ajustando las piezas que conforman el mosaico del "bien común", expresión de una justicia social que convierte un país en una buena sociedad. Algunos de los componentes de este bien común serían: el derecho al trabajo para todos, la seguridad social para todos, la renta básica mínima, la igualdad de oportunidades de acceso a la educación. Camps (1994) sostiene que además del Estado del Bienestar, el otro gran avance hacia la igualdad lo constituye el movimiento feminista.

No hay que confundir la igualdad material con el igualitarismo formal o jurídico. De lo que se trata es de concebir la igualdad material como punto de llegada, frente a la igualdad formal que es un punto de partida. Esto puede implicar, en determinados casos, el reconocimiento de derechos específicos para determinados colectivos que, por razones de raza, sexo y clase social están excluidos del reconocimiento efectivo de sus derechos fundamentales.

Cuales sean los criterios para determinar el contenido de la igualdad material es un asunto controvertido. Peces (1999) y Bobbio (1991) se inclinan por la satisfacción de las necesidades básicas como criterio para asegurar la igualdad material de todas las personas. Otros autores, como Añon (1994), discrepan de esta postura argumentando el carácter histórico social de las mismas que impide que todas ellas puedan cristalizar en derechos. Un intento de superación de esta discusión lo ofrecen los modelos de desarrollo a escala humana de Max-Neef y otros (1994) y la teoría de las necesidades humanas de Doyal y Gough (1994), que recurren al concepto de 
satisfactores que serían la manera histórica de satisfacer las necesidades humanas, las cuales sí que pueden considerarse universales.

El reconocimiento de derechos específicos variará si se trata de ciudadanos o no. En el primer grupo tendríamos a las minorías culturales o a las mujeres; en el segundo estarían los extranjeros. Para profundizar en el reconocimiento de derechos para las minorías puede consultarse Kymlicka (1996); para el reconocimiento de la igualdad en función del género, puede verse Kessier-Harris (1994), y, desde una perspectiva más filosófica, los trabajos de Valcárcer (1993) sobre igualdad y mujer (1993), las obras de Nussbaum, 2002). Para el tema de la condición de extranjero como limitadora de los derechos fundamentales, pueden consultarse los trabajos de De Lucas (1993; 1994; 1996; 1998; 2002).

Relacionada con la discusión sobre el igualitarismo, es importante destacar la reflexión que introduce el filósofo político contemporáneo Walzer. En su libro Las esferas de la justicia, Walzer (1993), distingue entre igualdad simple e igualdad compleja. La igualdad simple es la que pretende que todas las personas tengan la misma cantidad de las mismas cosas, sin tener en cuenta los esfuerzos y méritos deferentes de cada persona. Según Walzer, la justicia se halla en la igualdad compleja. Según este autor, la sociedad se compone de diversas esferas, cada una de las cuales sigue su propia lógica. La igualdad compleja es la situación resultante de repartir bienes de cada una de dichas esferas atendiendo, en cada caso, a la lógica de la esfera en cuestión (Siurana, 1998).

Por último, podemos destacar, por su vinculación con las teorías del desarrollo humano las aportaciones de Amartya Sen al concepto de igualdad. Sen (1995), retomando las críticas de Rawls al utilitarismo pero considerando insuficiente el planteamiento rawlsiano sobre los bienes primarios, plantea el concepto de igualdad de capacidades básicas, que vendría a combinar la idea de que las personas deben disponer de los bienes necesarios para poder desarrollar su vida, pero además deben tener las mismas capacidades para poder hacer uso de estos bienes.

\section{La responsabilidad}

Para Camps (1994), el objetivo de una educación para la libertad es formar personas responsables, personas que no necesiten siempre el agarradero de una norma escrita o el precepto moral, que aprendan a pensar por sí mismas y a explicar por qué actúan como actúan. El valor responsabilidad está estrechamente ligado con el de autonomía moral de la persona. Si ésta es la capacidad de escoger el principio adecuado a cada caso y procurarle dar la interpretación más justa, la primera es la capacidad que nos hace responsables de las acciones o de las omisiones, pues todo individuo tiene que responder ante sí mismo y ante los otros de lo que hace mal o de lo que podría hacer y no hace.

Hans Jonas, (1995), en un gran texto sobre El principio de responsabilidad afirma que, hoy más que nunca debido a los enormes avances tecnológicos que han contribuido al bienestar de muchas personas pero que también han generado grandes consecuencias negativas, es importante fomentar en las personas la responsabilidad por sus acciones. Jonas distingue entre responsabilidad causal, intencional, legal y ética. Una condición para la existencia de responsabilidad es el poder causal, es decir, que la acción u omisión sea la causa de algo; pero también se necesita que la responsabilidad sea intencional, es decir, que las consecuencias fueron presupuestas y buscadas. Otra distinción es la que existe ente responsabilidad legal y ética. La legal nos pide que al atender a las consecuencias de nuestras acciones 
tengamos en cuenta cuáles de ellas serán sancionadas legalmente, haya sido o no nuestra intención realizarlas. La responsabilidad ética nos pide que al atender a las posibles consecuencias de nuestras acciones tengamos en cuenta principios de justicia que deberían ser satisfechos, tengan o no un reconocimiento jurídico.

Según Siurana (1998), cuando atribuimos a una persona responsabilidad ética pensamos que la persona puede controlar su comportamiento a través de cuatro capacidades:

- a) Liberta de elección, es decir, capacidad de preferir una acción frente a otras posibles;

- b) Reflexión, capacidad de valorar racionalmente los motivos de su acción;

- c) Anticipación, capacidad de considerar las consecuencias previsibles de la acción;

- $\quad$ d) Sentido de la justicia, capacidad para distinguir lo justo de lo injusto.

Por lo general la responsabilidad se ejerce entre desiguales: la responsabilidad de los padres sobre el hijo, del maestro sobre el alumno, pero el objetivo debe ser siempre que cada persona pueda desarrollar sus capacidades para asumir sus propias responsabilidades. Como reflejo acertadamente Weber (1967), cuando mayor es el poder que una persona tiene, mayor es también su responsabilidad.

En efecto, todos somos en cierto grado corresponsables por las consecuencias de las acciones colectivas, por ejemplo, por la contaminación del medio ambiente, y todos tenemos que aportar algo de nuestra parte para solucionar los problemas comunes. Refiriéndose a esta responsabilidad por nuestras acciones en el futuro, Jonas (1995) defiende un nuevo imperativo categórico que formula de la siguiente manera: "Obra de tal manera que los efectos de tu acción sean compatibles con la permanencia de la vida humana en la tierra" (Jonas, 1995). A causa de la crisis ecológica producida por la dinámica tecnológica actual, Jonas defiende que se necesita una nueva ética de la responsabilidad para el futuro, cuyo principio cardinal sería: "preservar la permanente ambigüedad de la libertad del hombre, que ningún cambio de circunstancias puede jamás abolir, preservar la integridad de su mundo y de su esencia frente a los abusos del poder" (Jonas, 1995). De los efectos de la tecnología desde el punto de vista social y medioambiental se ocupan los estudios sobre Ciencia, Tecnología y Sociedad (CTS). El aspecto más innovador de este nuevo enfoque se encuentra en la caracterización social de los factores responsables del cambio científico. Se propone en general entender la ciencia-tecnología no como un proceso o actividad autónoma que sigue una lógica interna de desarrollo en su funcionamiento óptimo (resultante de la aplicación de un método cognitivo y un código de conducta), sino como un proceso o producto inherentemente social donde los elementos no epistémicos o técnicos (por ejemplo, los valores morales, las convicciones religiosas, los intereses profesionales, las presiones económicas) desempeñan un papel decisivo en la génesis y consolidación de las ideas científicas y los artefactos tecnológicos (Marino y otros, 2001). Con su principio de responsabilidad, Jonas (1995) realiza una crítica al principio de esperanza de Bloch (2004) ya que considera que la utopía del progreso transmitida por la modernidad es una de las tentaciones más peligrosas para el hombre. 


\section{El diálogo}

Desde Sócrates, en la tradición se tiene al diálogo como uno de los procedimientos más adecuados para encontrar la verdad, porque partimos de la convicción de que toda persona tiene al menos una parte de la verdad, que sólo dialógicamente puede salir a la luz (Platón, 1998).

La Ética Discursiva nace a comienzos de los años setenta en la Escuela de Frankfurt de la mano de Karl Otto Apel y Jürgen Habermas. Según Cortina (1989), la ética discursiva constituye la construcción filosófica propia de la etapa sexta en el desarrollo de la conciencia moral, tal y como Kohlberg lo expone, en la medida en que se basa en principios éticos universales, como la libertad, la igualdad, la solidaridad y la justicia, y adopta una perspectiva procedimental, basada en el diálogo, el cual deriva de la idea de competencia comunicativa del ser humano (Kohlberg, 1992). Según Apel, "todos los seres capaces de comunicación lingüística deben ser reconocidos como personas puesto que todas sus acciones y expresiones son interlocuciones virtuales" (Apel, 1985). La racionalidad en la que se basa la ética del discurso es una racionalidad discursiva, frente a la racionalidad lógicomatemática propia del mundo científico-técnico. Esta racionalidad sitúa al sujeto del conocimiento directamente en relación con el mundo objetivado, mientras que la segunda es autorreflexiva y dialógica. La ética del diálogo atiende a los intereses de las futuras generaciones que, aunque no puedan estar presentes en el momento de producirse esa situación ideal de habla, deberían contar con representantes para que pudieran hacerse oír sus argumentos.

Para que las soluciones dialogadas a los conflictos sean verdaderamente constructivas, los diálogos han de reunir una serie de requisitos señalados por la ética discursiva (Cortina, 1996):

1. El que toma el diálogo en serio ingresa en él convencido de que el interlocutor puede aportar algo, por eso está dispuesto a escucharlo;

2. No cree tener toda la verdad clara y diáfana y que el interlocutor es un sujeto hay que convencer, no con quien dialogar. Un diálogo es bilateral no unilateral;

3. Está dispuesto a escuchar para mantener su posición si no le convencen los argumentos del interlocutor, o a modificar si le convencen;

4. Está preocupado por buscar una solución correcta y, por tanto, por entenderse con el interlocutor. Entenderse no significa conseguir un acuerdo total, pero si descubrir todo aquello que ya tenemos en común;

5. La decisión final ha de atender intereses universalizables, es decir, los de todos los afectados.

El diálogo con estas condiciones se produce en la situación ideal de habla de Habermas (1985) o en la comunidad ideal de comunicación de Apel (1991). En este escenario, todos los seres humanos que puedan armonizarse con las necesidades de los demás por vía argumentativa. No se trata de una comunidad utópica, al estilo de la de Tomás Moro, sino que se trata de una idea regulativa al estilo kantiano, para medir la altura ética de las sociedades actuales por lo próximas o alejadas que estén de dicha idea. En esta comunidad, se distribuyen simétricamente las oportunidades de elegir y realizar actos de habla y se garantiza que los roles de diálogo sean intercambiables. Ello representa una forma de diálogo y de vida ideal, 
que sirve como crítica de los consensos fácticos, puesto que reúne los requisitos que debería cumplir un consenso racional.

Por referencia a la comunidad ideal, es posible criticar el que en la vida real no haya auténticos diálogos. Por eso, la ética del discurso defiende que es preciso aumentar el nivel material y cultural de los sujetos, para que nadie sea excluido de los diálogos donde se tratan temas que les afectan y puedan dialogar en condiciones de simetría.

¿Tolerancia o respeto activo?

Uno de los valores morales propios de la tradición liberal ha sido el de la tolerancia. Para Camps (1994), hay tres grandes grupos que señalan las diferencias que han producido y siguen produciendo intolerancia:

- Diferencias de creencias y opiniones. Al primer grupo pertenecen las diferencias ideológicas y las religiosas. Los primeros alegatos a favor de la tolerancia proviene de los filósofos de los siglos XVII y XVIII que atacaban la pretensión de las Iglesias monoteístas de ser depositarias de la verdad religiosa y representar al Dios único y verdadero. Lo que se reivindicaba por autores como Locke o Voltaire es que las religiones no se basan en verdades sino en creencias, y que todas ellas son igualmente legítimas.

- Diferencias económicas. El segundo grupo lo constituyen las diferencias económicas, que derivan de las diferencias sociales y culturales que provocan rechazo. Pero argumenta Camps (1994) aunque se apele a diferencias de carácter étnico, al inmigrante o al gitano no se les tolera no porque pertenezca a otra cultura, sino porque su presencia significa pobreza, marginación, inseguridad, desorden e incluso es muestra de una injusticia que hay que tomarse la molestia de encubrir o resolver. Al gitano o al árabe rico no se le margina. Se margina al desposeído porque su presencia incomoda o no agrada.

- Diferencias físicas. El tercer grupo lo constituyen las diferencias físicas o fisiológicas, donde entraría la intolerancia hacia los minusválidos, los retrasados mentales, los homosexuales, aunque a este último grupo se le ha rechazado durante siglos al amparo de las doctrinas religiosas.

Tradicionalmente, se ha diferenciado entre dos formas de tolerancia: la tolerancia negativa, es decir la neutralidad en el sentido de la no intervención dentro de las diferentes formas individuales y/o socioculturales de vida, y la tolerancia positiva, que considera un deber apoyar a todas ellas en la búsqueda de sus ideales de vida dentro de los límites de la igualdad de oportunidades para todos.

Sin embargo, estamos de acuerdo con De Lucas (1996) cuando argumenta que la tolerancia, no como virtud privada sino como concepto normativo, no es un principio jurídico-político válido. Lo que se denomina tolerancia viene suplido con ventaja por la igualdad en las libertades, por el reconocimiento de la titularidad y el ejercicio de los derechos. La tolerancia liberal tolera la diferencia en aras de la estabilidad, pero no reconoce que el diferente pueda ser sujeto de los mismos derechos. Según De Lucas (1996) los problemas de tolerancia a los que no puede hacer frente la tradición liberal surgen cuando diferentes prácticas, estilos de vida, identidades minoritarias que presentan problemas de discriminación, de exclusión, de invisibilidad, de opresión, reclaman carta de ciudadanía en el ámbito público. De Lucas reclama la interculturalidad, es decir, la participación de todos los grupos 
presentes en nuestras sociedades en la discusión y reelaboración de un consenso, desde una mutua actitud de responsabilidad y respeto por la autonomía, pero también por las reglas del juego, reglas que estarían constituidas por las reglas de juego de la democracia y por el respeto a los derechos humanos. Se trata, por tanto, de pasar de tolerar la diferencia a practicar un respeto activo, que parte del interés por comprender a los otros y por ayudar a llevar adelante sus planes de vida.

Al igual que veíamos en relación con el diálogo, también el respeto activo debería ser un elemento clave en la educación, la apreciación de cada persona es diferente pero igualmente digna y que hemos de aprender de unos de los otros en un principio básico en el trabajo en una cooperación y educación comprometida con la defensa de los derechos humanos

\section{La solidaridad}

La solidaridad constituye una versión secularizada del valor fraternidad, que es el tercero de los que defendió la Revolución Francesa, junto a la libertad y la igualdad. Si la fraternidad hacía referencia a que todos los seres humanos somos hijos de un mismo padre, la solidaridad suprimirá el aspecto religioso de esta idea, pero mantendrá la idea de sentirse unido al resto de la humanidad.

El itinerario histórico de la solidaridad, diferencia la denominada solidaridad de los antiguos de la solidaridad de los modernos. Según Peces (1999), la primera se centra en una dimensión ética y religiosa (y que tiene en opinión del autor cuatro elementos comunes: el vínculo de amistad, un objetivo de comunidad, un uso común de bienes y una ayuda mutua), mientras que la segunda se constituye en un principio jurídico-político. Los teóricos aparecen a partir de la Revolución Francesa y tienen sus máximos exponentes en las doctrinas del socialismo utópico y del anarquismo.

Según Camps ( 1994), la solidaridad es el complemento de la justicia, es el sentimiento de solidaridad el que ha de llevarnos, por una parte, a denunciar las injusticias y, por otra, a compensar las insuficiencias de la justicia. En primer lugar, sin sentimientos solidarios, es difícil que se luche contra las injusticias ya que los desposeídos y marginados, los que sufren más la falta de justicia, carecen de voz y sólo pueden hacerse oír a través de alguien que les escuche y que, de manera solidaria, habla en su nombre. En segundo lugar, la justicia se materializa en las leyes, y éstas no suelen atender a las diferencias individuales, sino a lo que iguala a las personas. La ley $\mathrm{u}$ acción institucional, general por definición, no tiene capacidad para atender a la especificidad del sufrimiento particular, mientras que la solidaridad, como virtud privada, puede promover actitudes de corresponsabilidad frente a problemas que deben afectarnos a todos porque son de toda la sociedad. Es en esta actitud de lucha contra las injusticias donde creemos que reside la diferencia entre caridad y solidaridad. La primera es una acción puntual para dar a otros algo que nos sobra, mientras que el que es solidario reflexiona sobre las injusticias -políticas, económicas o de otro tipo- y realiza acciones dirigidas a erradicarlas.

De Lucas (1993) plantea dos objeciones a la solidaridad como principio ético: la primera es que la solidaridad no puede darse en una sociedad cerrada. Aunque exista comunión, reconocimiento de similitudes entre los miembros de un grupo criminal, o racista, no estaríamos hablando de solidaridad puesto que ésta no puede ser a la vez auténtica solidaridad y excluyente. La segunda se refiere a las condiciones de subsistencia del principio de solidaridad en una sociedad individualista y atomizada, como la nuestra. 
La constatación de que en las sociedades occidentales prima el valor de lo singular, de la individual. Puede verse el trabajo de Zubero (1994), donde se apunta que el desarrollo del capitalismo ha supuesto, en primer lugar, un proceso de colonización de todo el mundo de la vida por la racionalidad económica a la par que de disolución de cualquier control moral de esa racionalidad y, en segundo lugar, un proceso de individualización de la sociedad, haciendo retroceder las estructuras comunitarias al cada vez más restringido espacio de la privacidad, despojándolas de toda relevancia pública.

Superar estas objeciones no es una tarea en absoluto sencilla, pero, existen problemas de alcance global que hacen, más que nunca, necesario el principio de solidaridad. Peces (1999) le atribuye una función muy valiosa al principio de solidaridad ya que, permitiendo un diálogo ilustrado entre personas que se respetan y se reconocen y que contribuyen, en ese esquema compartido, a poner en común, a discutir y a formar criterios morales que superan la pura individualidad. En este sentido, la solidaridad aportaría esa perspectiva de pertenencia a una comunidad mundial al valor diálogo, incorporando la consideración de todos los afectados por las acciones. Otra de las aportaciones del principio de solidaridad es que provee de fundamentación a los derechos de tercera generación (derecho al desarrollo, a la paz, al medio ambiente). Además, a partir de la idea de humanidad, una de las claves de la solidaridad, se puede defender la idea de todos los hombres deben estar en igualdad en la repartición de derechos y deberes básicos.

Por último, el valor solidaridad es un instrumento clave para la comprensión de la aplicación y para la interpretación de los derechos. Tanto los titulares de los derechos como los operadores jurídicos encargados de su protección y garantía pueden inclinarse por un uso egoísta o un uso solidario de los mismos.

\section{La justicia}

En la Declaración Universal de los Derechos Humanos, el valor justicia aparece ligado a los valores de liberta e igualdad, pero, desde algunas otras posiciones teóricas (Capms, 1990, Cortina, 1994) la justicia se configura como el núcleo de la ética, englobando todos los demás valores. No sería la postura que defiende, entre otros autores, Heller (1990), quien afirma que la ética no siempre acaba en la justicia sino que puede ir más allá. Hay determinadas acciones como la fidelidad al amigo, el acercamiento al enfermo, el apoyo al perseguido, que no son exigidas sólo por la justicia sino por el reconocimiento del otro como igual a nosotros. Es, por tanto, un valor ampliamente tratado y discutido desde muchas aproximaciones teóricas. De entre ellas, hemos seleccionado tres contribuciones:

1) La de Rawls (1978), probablemente la más completa teoría que se haya formulado sobre este valor, posteriormente ha habido las propuestas de Sen (1995) y Nussbaum (2002).

2) La idea de justicia de la ética discursiva de Apel (1985) y Habermas (1985), porque nos remite nuevamente al valor diálogo

3) La de la teología de la liberación de Dussel (1986), esta última muy conectada con el discurso sobre el desarrollo. Somos conscientes que podríamos enumerar las muchas aproximaciones a la idea de justicia desde la antigüedad clásica.

De entre las teorías actuales sobre la justicia, queremos destacar los principales rasgos de La Teoría de la Justicia de Rawls (1978). Rawls realiza una crítica al 
utilitarismo que sostiene que las decisiones son más justas cuando logran la mayor felicidad del mayor número de personas. Rawls afirma que, si se siguen las tesis utilitaristas, puede que haya personas que no lleguen a disfrutar de esa felicidad porque no entran en la mayoría. Por ello, lo que sí que tiene que asegurarse en una sociedad bien ordenada son estos tres tipos de justicia:

1) Libertades básicas, compatibles con las libertades de los demás

2) Igualdad de oportunidades

3) El principio de la diferencia, según el cual, el Estado debe distribuir los bienes primarios de tal manera que favorezca a quienes más lo necesiten. (Los bienes primarios son medios de uso general que ayudan a todo el mundo a promover sus fines, y comprenden los derechos, las libertades y las oportunidades, la renta y la riqueza y las bases sociales del respeto a uno mismo).

El sujeto de la justicia lo constituyen las instituciones democráticas, y es responsabilidad del Estado traducir los principios de justicia en políticas públicas concretas.

Las propuestas de Apel (1991) y Habermas (1985), defensores de la ética del diálogo, en relación con la idea de justicia. Según estos autores, una acción es justa si es aceptada sin coacción por todos los afectados tras un diálogo racional en condiciones de simetría. Las condiciones en las que se producen los diálogos en la actualidad no son racionales (no existe en la realidad la situación ideal de habla), sino que esta comunidad ideal de diálogo nos sirve de horizonte de justicia, pudiendo medir en qué grado las sociedades son más o menos justas por lo próximas o alejadas que se encuentren de realizar la situación ideal. En los Estados en los cuales los ciudadanos son sistemáticamente excluidos de la toma de decisiones o son perseguidos a causa de sus ideas, sería justo ocultar información e incluso hacer uso de un mínimo de violencia para salvar la propia vida. Pero, sugiere Siurana (2001), la justicia exige que se haga únicamente uso de la violencia cuando sea necesario para la conservación, y se ponga el énfasis en las relaciones pacíficas y el diálogo transparente, teniendo en cuenta que en la situación de justicia perfecta el uso de la violencia sería definitivamente eliminado.

La propuesta de Dussel (1986) defensor de La Ética de la Liberación. En un mundo dominado por las injusticias, donde se constatan los fracasos del desarrollismo y donde el sistema capitalista se impone como el único modelo económico de referencia, la principal tarea de la ética de la liberación es desfondar, perforar el fundamento del sistema hacia otro fundamento más allá o trascendental al presente sistema. La ética de la liberación exige repensar la totalidad de los problemas morales desde la perspectiva y las exigencias de la responsabilidad por el pobre.

\section{La paz}

En la Declaración Universal de los Derecho Humanos, la paz aparece como uno de los valores guía junto con la libertad y la justicia. El valor paz, a juicio del investigador, presenta una cierta complejidad reivindicada desde el ámbito de los estudios sobre la paz. Así, se suelen distinguir dos concepciones diferentes de entender la paz: la paz negativa y la paz positiva. Según Lederach (2000), uno de los mayores expertos en la investigación y estudio sobre la paz, las características fundamentales de la concepción de paz negativa, que es la predominante en la actual comprensión contemporánea del término, son las siguientes: 
- La paz es fundamentalmente un concepto negativo. Se define como la ausencia de violencia (bélico/explotación) o como el estado en tiempo de no-guerra. Es la ausencia de una 'condición anhelada'. Por eso es un concepto muy limitado, que se ha definido cada vez más en función del fenómeno de guerra y del hecho bélico, hasta el extremo de que, fuera de su contraste con la guerra, la paz carece de contenido palpable.

- Debido a que la estructura Occidental (social, cultural y política) es la dominante en el mundo de hoy, también prevalece su concepción de la paz. La paz se concibe en función de dos fenómenos. Por una parte es asimilada al mantenimiento de la unidad y el orden interior (que beneficia normalmente a los intereses dominantes), con la preparación defensiva exterior. Por otro lado, sólo el Estado nación tiene derecho a usar la fuerza, es decir, la paz es materia y competencia exclusiva de los Estados.

- Esta concepción de la paz ha sido potenciada y propagada por la educación. Normalmente la educación otorga un papel dinámico e importante a la guerra y a la formación de la política internacional actual. La paz, en cambio, queda reducida a un proceso estático y poco potente, como una especie de intermedio entre actos en la obra maestra que es la historia.

- La paz, hoy día, está limitada al concepto de paz en sentido de pactos, dominación interior y fuerte preparación militar exterior. Refleja los intereses de los que se benefician de la estructura internacional tal y como es, es decir, los del centro, no los de periferia.

Frente a esta concepción limitada de la paz, Lederach (2000) propone el concepto de paz positiva. Esta manera de entender la paz se concretaría de la siguiente manera: en primer lugar, la paz se entendería como un valor, un concepto rico y multidimensional que trasciende la idea de paz como una mera ausencia de guerra, ni como un 'asunto de gobierno' a escala internacional. Por ello, la paz se entiende cómo la liberación del individuo de todo lo que le impide gozar de los elementos de la vida, sea debido a la violencia directa (homicidio o guerra) o a la violencia estructural (racismo, hambre, marginación).

Asimismo, la paz se asimila a un orden de reducida violencia y elevada justicia (Bobbio, 1991). La paz requiere justicia social, sin que exista superioridad, dominio o marginación. Sí que debe existir igualdad en cuanto al control y distribución del poder y de los recursos, la paz es un proceso, no un concepto estático. Finalmente, la paz es también la presencia de condiciones y circunstancias deseadas. La paz positiva es la colaboración, la cooperación, el mutuo entendimiento, la confianza. Es una asociación activa, caracterizada por el mutuo beneficio de una relación positiva.

Desde un punto de vista más realista, la idea de paz positiva dista mucho de ser realidad. Como recuerda Camps (1994), la guerra no ha dejado de ser una realidad natural para la existencia humana y la industria armamentística es una parte imprescindible de la organización política y económica de todas las naciones. Por ello, son de tremenda actualidad las reflexiones que en 1795 hacía Kant a propósito de la paz en su ensayo Sobre la paz perpetua, en el que sostenía que la paz no es un estado natural en las organizaciones humanas, sino algo qu debe ser instaurado. La paz es un deber, porque la vida es un derecho de todos los humanos. 


\section{CONCLUSIONES}

Hacer referencia a la dimensión axiológica de la educación como una estrategia de cooperación al desarrollo significa explicitar qué sistema valorativo marco asumimos desde nuestro enfoque. Nos parece extremadamente importante poner el centro en la dimensión axiológica por varias razones: en primer lugar porque como afirmaba Aranguren (1993) en su Ética (1958; 1993), toda persona sea buena o mala y antes de serlo es forzosamente moral, por ser inteligente y libre. Moral en el sentido de la moral como estructura porque tiene que justificar su vida, en su totalidad y, acto por acto, en cada una de sus partes. Y también en el sentido de la moral como contenido, porque el ser humano es libre y tiene que elegir cuál va a ser su bien, de realizarlo en el mundo y de apropiárselo personalmente. No existe el hombre biológico, desnudo de cultura, de valores, parece, pues, incuestionable, que estamos llamados a recorrer ese camino, a cuestionarnos lo que nos rodea, a valorar y actuar en consecuencia.

Además porque tanto en el ámbito de la educación como en el del desarrollo, se refleja el carácter moral del ser humano. Por lo que respecta al campo de la educación la condición moral se manifiesta tanto en la actuación profesional como en los contenidos educativos; como sugieren Ortega y Mínguez (2001), en cualquier actuación profesoral estamos filtrando y proyectando una determinada concepción de la persona, promoviendo unos determinados valores, por lo mismo que no podemos renunciar a nuestra condición de humanos que viven y actúan desde unos valores.

\section{REFERENCIAS BIBLIOGRÁFICAS}

Añon, M. (1994). "Las Necesidades y el Fundamento de los Derechos Humanos", Centro de Estudios Constitucionales, Madrid.

An-Na'lm, A. (1992). "Human Rights in Cross-Cultural Perspectives. A Quest for Consensus”, Philadelphia, University of Pennsylvania Press.

An-Na'lm, A. (1999). "Expanding the limits of Imagination: Human Rights from a Participatory Approach to Multilateralism". Innovation in Multilateralism. Tokyo, New York, Paris, United Nation University Press.

Apel, K. (1985). "La transformación de la Filosofía”, vol. 2, Taurus, Madrid.

Apel, K. (1991). “Teoría de la Verdad y Ética del Discurso”, Paidós, Barcelona.

Aranguren, J. (1993). “Ética”, Alianza Universidad Textos, Madrid.

Bloch, E. (2004). “El principio esperanza”. Trolla, Madrid.

Bobbio, N. (1991). "El tiempo de los derechos”, Sistema, Madrid.

Camps, V. (1994). "Los valores en la educación”, Alauda/Anaya, Madrid.

Constant, B. (1989). "Escritos políticos", Centro de Estudios Constitucionales, Madrid. 
LOS VALORES EN EDUCACIÓN PARA EL DESARROLLO

Cortina, A. (1989). "La ética discursiva" en Camps, V. (ed.), Historia de la ética, vol. III, Crítica, Barcelona.

Cortina, A. (1994). "La ética de la sociedad civil”, Anaya, Madrid.

Cortina, A. (1996). "Educar moralmente ¿Qué valores para qué sociedad?”.

De Lucas, J. (1993). “El concepto de solidaridad”, Fontamara, México.

De Lucas, J. (1994). “El desafío de las fronteras”, Temas de Hoy, Madrid.

De Lucas, J. (1996). “Tolerancia y derecho, ¿Tiene sentido hablar de tolerancia como principio jurídico?”, Isegoría, 14.

De Lucas, J. (1998). "La globalización no significa universalidad en los derechos humanos”, Jueces para la democracia, 32.

De Lucas, J. (2002). "El vínculo social, entre ciudadanía y cosmopolitismo”. El vínculo social: Ciudadanía y cosmopolitismo, Tirant lo Blanch, Valencia.

Doyal, L. y Gough, I. ( 1994). “Teorías de las necesidades humanas”, Icaria, Barcelona.

Dussel, E. (1986). "La ética de la liberación”, Ediciones Paulinas, Madrid.

Giner, S. (1989). "Sociología y filosofía moral” en Camps, V. (ed.), Historia de la ética, vol. III., Crítica, Barcelona.

Habermas, J. (1985). "Conciencia moral y acción comunicativa”. Península, Barcelona.

Howard, R. (1993). "Cultural absolutism and the nostalgia of the community", Human Rights Quarterly, 15.

Jonas, P. (1995). “El principio de responsabilidad”, Herder, Barcelona.

Kant, I. (1992). “Fundamentación de la Metafísica de las Costumbres”, Real Sociedad Económica Matritense de Amigos del País, Madrid.

Kessier-Harris, A. (1994). "Feminism and affirmative action" en Mills, N. (ed.) Debatting affirmative Action, Delta Books, New York.

Kohlberg, L. (1992). “Psicología del desarrollo moral”, Desclée de Brower, Bilbao.

Kymlicka, W. (1996). “Ciudadanía multicultural”, Paidós, Madrid.

Lederach, J. (2000). “El Abecé de la paz y los conflictos. Educación para la paz”, Los Libros de la Catarata, Madrid.

Marino, E., González, J., López, J., Martín, M., Osorio, C. y Valdés, C. (2001). "Ciencia, Tecnología y Sociedad: una aproximación conceptual", Cuadernos de Iberoamérica, OEI, Madrid. 
Martínez, E. (2000). “Ética para el desarrollo de los pueblos”, Trotta, Madrid.

Max-Neef. M., Elizalde, A. y Hopenhayn, M. (1994). "Desarrollo a escala humana”, Icaria, Barcelona.

Monzón, A. (1992). “Diálogo intercultural y derechos humanos” en Ballesteros, J. (ed.), Los Derechos Humanos, Tecnos, Madrid.

Nussbaum, M. (1998). "Capacidades humanas y justicia social. En defensa del esencialismo aristotélico" en Riechmann, J. (coord..), Necesitar, Desear, Vivir. Sobre necesidades, desarrollo humano, crecimiento económico y sustentabilidad, La Catarata, Madrid.

Nussbaum, M. (1999). "Patriotismo y cosmopoltismo" en Nussbaum, M. (ed.), Los Límites del Patriotismo. Identidad, Pertinencia y "Ciudadanía Mundial”, Paidós, Barcelona.

Nussbaum, M. (2002). "Las mujeres y el desarrollo humano. El enfoque de las capacidades", Herder, Barcelona.

Ortega, P. y Mínguez, R. (2001). "Los valores en la educación”, Ariel Educación, Madrid.

Payá, M. (1997). "Educación en valores para una sociedad abierta y plural: Aproximación conceptual”, Desclé de Brower, Bilbao.

Peces, G. (1999). “Curso de Derechos Fundamentales”, BOE, U. Carlos III, Madrid.

Pérez, J. (1996). "Claves humanistas para una educación democrática”, Alauda Anaya, Madrid.

Petrella, R. (1997). "El Bien común. Elogio de la solidaridad”, Temas de Debate, Madrid.

Platón (1998). “El sofista, Diálogos V”, Gredos, Madrid.

Rawls, J. (1978). “Teoría de la Justicia”. Fondo de Cultura Económica, Madrid.

Sen, A. (1995). "Nuevo examen de la desigualdad”, Alianza, Madrid.

Siurana, J. (1998). "Historia de la idea de justicia” en Cortina, A. \& Col., Educar en la justicia, Generalitat Valenciana, Valencia.

Thiebaut, C. (1999). “Tres tensiones de nuestra moral ciudadana”, Leviatán, 75.

Walzer, M. (1993). "Las esferas de la justicia. Una defensa del pluralismo y de la igualdad”. Fondo de Cultura Económica, México.

Weber, M. (1967). "El político y el científico”. Alianza, Madrid.

Zubero, I. (1994). "Las nuevas condiciones de la solidaridad”. Desclée de Brower, Bilbao. 